1908.] SINGULAR POINTS OF A DIFFERENTIAL EQUATION. 223

\title{
SINGULAR POINTS OF A SIMPLE KIND OF DIFFERENTIAL EQUATION OF THE SECOND ORDER.
}

\author{
BY PROFEsSOR C. A. NOBLE.
}

(Read before the San Francisco Section of the American Mathematical Society, September 28, 1907.)

In a series of four memoirs in the Journal de Mathématiques (series 3 , numbers 7,8 ; series 4 , numbers 1, 2) Poincaré has, among other things, discussed the topology of curves defined by ordinary differential equations of a simple character. In a recent course of lectures Hilbert laid considerable stress on the importance of these results and exhibited an elegant method for obtaining them in the case of a differential equation of the form $d y / d x=(c x+d y) / a x+b y)$. In the following paper I have shown how the same method can be used for an ordinary differential equation of the second order. My results tally with those of Poincaré insofar as the latter are enumerated; but they are more detailed than his and are, I think, more simply obtained.

Given (1) $d^{2} y / d x^{2}=(d x+e y+f \cdot d y / d x) /(a x+b y+c \cdot d y / d x)$, $a, b, c, d, e, f$ real constants. Put. (2) $d y / d x=z$ and (1) becomes

$$
d z / d x=(d x+e y+f z) /(a x+b y+c z) .
$$

Now write

(4) $d x / d t=a x+b y+c z, \quad d y / d t=z, \quad d z / d t=d x+e y+f z$.

Multiply the second and third of these equations by $m$ and $n$ respectively and add,

(5) $d(x+m y+n z) / d t=(a+n d) x+(b+n e) y+(c+m+n f) z$.

Equate the second member of $(5)$ to $\lambda(x+m y+n z)$ and determine $m, n$ and $\lambda$ accordingly. We find

$$
\begin{gathered}
n=(\lambda-a) / \lambda, \quad m=(b d-a c+e \lambda) / d \lambda \\
\lambda^{3}-(a+f) \lambda^{2}+(a f-c d-e) \lambda-(b d-a e)=0 .
\end{gathered}
$$


224 Singulaar points of a differential equation. [Feb.,

Indicate the three values of $\lambda$ arising from (8) and the corresponding values of $m, n$ by subscripts. Put

9) $x+m_{1} y+n_{1} z=\xi, \quad x+m_{2} y+n_{2} z=\eta, \quad x+m_{3} y+n_{3} z=\zeta$

and we obtain as the equivalent of (4)

$$
d \xi / d t=\lambda_{1} \xi, \quad d \eta / d t=\lambda_{2} \eta, \quad d \zeta / d t=\lambda_{3} \zeta
$$

or, eliminating $t$,

(11) $d \eta / d \xi=\kappa \cdot \eta / \xi, d \zeta / d \xi=\mu \cdot \zeta / \xi$, where $\kappa=\lambda_{2} / \lambda_{1}, \mu=\lambda_{3} / \lambda_{1}$.

The problem in hand is to investigate the nature of the critical point $(0,0,0)$ in $x, y, z$ space for the equations (4). By virtue of the substitutions (9) this point goes over into $(0,0,0)$ in $\xi, \eta, \zeta$ space. There are three principal cases to consider, depending upon the roots of (8). They are $:$ I. The $\lambda_{i}$ all real and different; II. Two $\lambda_{i}$ conjugate complex; III. 'Two or more $\lambda_{i}$ identical.

I. $\lambda_{1}, \lambda_{2}, \lambda_{3}$ real and different. The integrals of (11) are

$$
\eta=C \xi^{\kappa}, \quad \zeta=D \xi^{\mu} \quad(C, D \text { integration constants }) .
$$

The intersections of these two surfaces constitute $\infty^{2}$ integral curves. How are these curves distributed in the immediate vicinity of $(0,0,0)$ ? How many pass through $(0,0,0)$ ? An answer to these questions amounts to a characterisation of the critical point. There are three possibilities. 'They arise according as $\kappa, \mu$ are (i) both $>0$, (ii) both $<0$, or (iii) unlike in sign.

(i) $\kappa>0, \mu>0$. By appropriately naming the roots of (8) we can make $\kappa>1, \mu>1$. Every cylinder (12) passes through the $\zeta$-axis and is tangent to the plane $\eta=0$. Every cylinder (13) passes through the $\eta$-axis and is tangent to the plane $\zeta=0$. There are therefore $\infty^{2}$ integral curves passing through $(0,0,0)$, all tangent to one of their number, viz., to the $\xi$-axis. Hilbert would probably call this singular point a Scheitel-scheitelpunkt.

(ii) $\kappa<0, \mu<\Omega$. We may write (12), (13) in the form

$$
\eta \xi^{-\kappa}=C, \quad \zeta \xi^{-\mu}=D .
$$

The only integral curves passing through $(0,0,0)$ now are $\xi=0=\eta, \eta=0=\zeta$, and $\zeta=0=\xi$. (12) represents a family of hyperbolic cylinders with generators parallel to the 
$\zeta$-axis; (13) a family of hyperbolic cylinders with generators parallel to the $\eta$-axis. All the $\infty^{2}$ integral curves, barring the three above mentioned, tend to hug the $\xi$-axis and the $\eta \zeta$-plane. Hilbert would call this singular point a Sattel-sattelpunkt.

(iii) $\kappa>0, \mu<0$ (or $\kappa<0, \mu>0$ ). $W$ rite (12), (13) in the form

$$
\eta=C \xi^{\kappa}, \quad \zeta \xi^{-\mu}=D
$$

Every cylinder (12) yields two integral curves through $(0,0,0)$, viz., the curves of intersection with $\xi=0$ and with $\zeta=0$ (these two planes are members of (13)). There are thus $\infty^{1}$ integral curves through the origin, all of them lying in one plane, except one, this one being the $\zeta$-axis. Hilbert would probably call this singular point a Scheitel-sattelpunkt mit isoliertem Strahl.

II. $\lambda_{1}$ real; $\lambda_{2}, \lambda_{3}$ conjugate complex. The integrals of (10) may now be written

$$
\begin{gathered}
\eta=C \xi^{\kappa}, \quad \zeta=D \eta^{\nu} \\
\left(\kappa=\lambda_{2} / \lambda_{1}, \nu=\lambda_{3} / \lambda_{2}, C, D \text { arbitrary constants }\right) .
\end{gathered}
$$

If we seek an interpretation of (12), (14) in real space by means of the substitutions

$$
\xi=x+m_{1} y+n_{1} z=X,
$$

$$
\begin{aligned}
& \eta=x+m_{2} y+n_{2} z=x+m_{2}^{\prime} y+n_{2}^{\prime} z+i\left(m_{2}^{\prime \prime} y+n_{2}^{\prime \prime} z\right)=Y+i Z, \\
& \zeta=x+m_{3} y+n_{3} z=x+m_{2}^{\prime} y+n_{2}^{\prime} z-i\left(m_{2}^{\prime \prime} y+n_{2}^{\prime \prime} z\right)=Y-i Z
\end{aligned}
$$

whereby

$m_{2}=m_{2}^{\prime}+i m_{2}^{\prime \prime}, m_{3}=m_{2}^{\prime}-i m_{2}^{\prime \prime}, n_{2}=n_{2}^{\prime}+i n_{2}^{\prime \prime}, n_{3}=n_{2}^{\prime}-i n_{2}^{\prime \prime}$, we get from (12)

$$
\begin{aligned}
& Y+i Z=C X^{\lambda_{2} / \lambda_{1}}=C X^{\lambda_{2}^{\prime} / \lambda_{1}} \cdot e^{i\left(\lambda_{2}^{\prime \prime} / \lambda_{1}\right) \log X} \\
& =C X^{\lambda_{2} / \lambda_{1}}\left\{\cos \left[\left(\lambda_{2}^{\prime \prime} / \lambda_{1}\right) \log X\right]+i \sin \left[\left(\lambda_{2}^{\prime \prime} / \lambda_{1}\right) \log X\right]\right\} \\
& \left(\lambda_{2}=\lambda_{2}^{\prime}+i \lambda_{2}^{\prime \prime}\right) .
\end{aligned}
$$


226 Singular PoINTS of a differential Equation. [Feb.,

Separating real and imaginary parts in (16) and equating,

$$
\begin{aligned}
& Y=C X^{\lambda_{2} / \lambda_{1}} \cos \left[\left(\lambda_{2}^{\prime \prime} / \lambda_{1}\right) \log X\right], \\
& Z=C X^{\lambda_{2} / \lambda_{1}} \sin \left[\left(\lambda_{2}^{\prime \prime} / \lambda_{1}\right) \log X\right] .
\end{aligned}
$$

Squaring (17), (18) and adding we obtain

$$
Y^{2}+Z^{2}=C^{2} X^{2 \lambda_{2}{ }^{\prime} / \lambda_{1}}
$$

a surface, or rather $\infty^{1}$ surfaces, of revolution upon which all the $\infty^{2}$ integral curves lie. Again, we have from (14) by means of (15),

$$
Y-i Z=D(Y+i Z)^{\nu} .
$$

Let us introduce polar coördinates into the $Y, Z$ plane by putting $Y+i Z=\rho e^{i \phi}$. We obtain from (20)

$$
\begin{array}{r}
\rho=\text { constant } \cdot e^{i \phi(1+\nu) /(1-\nu)} \\
=\text { constant } \cdot e^{\phi \lambda_{2}^{\prime} / \lambda_{2}^{\prime \prime}}=e^{\left(\lambda_{2}^{\prime} \mid \lambda_{2} 2^{\prime \prime}\right)(\phi+\text { const. }),}
\end{array}
$$

a family of $\infty^{1}$ spiral cylinders winding about the $X$-axis. The $\infty^{2}$ integral curves are thus the intersections of the two sets of surfaces in $X, Y, Z$ space

$$
\begin{aligned}
& Z=C X^{\lambda_{2}^{\prime} \lambda_{1}} \cdot \sin \left[\left(\lambda_{2}^{\prime \prime} / \lambda_{1}\right) \log X\right], \\
& \quad \rho=e^{\left(\lambda_{2} / \lambda_{2}{ }^{\prime \prime}\right)\left(\phi+C^{\prime}\right)} \quad\left(C^{\prime} \text { an arbitrary constant }\right) .
\end{aligned}
$$

The critical point $(0,0,0)$ in $X, Y, Z$ space, which is likewise the origin in $x, y, z$ space, might be called a Strudelpunkt, by analogy with the corresponding situation which arises for a differential equation of the first order. The surfaces (18) lie entirely in space $X>0$, and they all contain the $Y$-axis as a "stop line" when $\lambda_{2}^{\prime} / \lambda_{1}>0$. If $\lambda_{2}^{\prime} / \lambda_{1}>0$, none of the surfaces (18) (except the one for $C=0$ ) contains the $Y$-axisthey cease being defined when $X$ approaches zero. In fact, these cylinders (18) have, along the $Y$-axis, a definite tangent plane only when $\lambda_{2}^{\prime} / \lambda_{1}>1$. In the present case, therefore, there is no integral curve containing $(0,0,0)$. If, however, $\lambda_{2}^{\prime} / \lambda_{1}>1$, there will be $\infty^{2}$ curves which approach $(0,0,0)$ asymptotically in space $X>0$.

For the topological discussion of the equation of the first order there is but one integral equation, viz., the analogon of 
(21), and the special assumption $\lambda_{2}^{\prime} / \lambda_{2}^{\prime \prime}=0$ then yields the special class of singular point called Wirbelpunkt, which is completely enclosed by each one of the $\infty^{1}$ integral curves $\rho=$ constant. Here, however, in space, no such situation can arise, because (18) loses geometric meaning for $\lambda_{2}^{\prime} / \lambda_{2}^{\prime \prime}=0$. There appears to be nothing in the present problem analogous to the Wirbelpunkt in the plane.

III $_{1} . \lambda_{3}=\lambda_{2} \neq \lambda_{1}$. The three equations (10) now shrink to two, and another means of integrating (4) must be devised. Take the three equations

$$
d \xi / d t=\lambda_{1} \xi, \quad d \eta / d t=\lambda_{2} \eta, \quad d z / d t=d x+e y+f z .
$$

Assume the second member of the third equation identically equal to $\alpha \xi+\beta \eta+\gamma z$. This necessitates

$$
\alpha=\lambda_{1} d /\left(\lambda_{1}-\lambda_{2}\right), \quad \beta=-\lambda_{2} d /\left(\lambda_{1}-\lambda_{2}\right), \quad \gamma=\lambda_{2},
$$

so that, instead of (10), we have as our three equations

$$
\begin{aligned}
& d \xi / d t=\lambda_{1} \xi, \quad d \eta / d t=\lambda_{2} \eta, \\
& d z / d t=\left(\lambda_{1} d /\left(\lambda_{1}-\lambda_{2}\right)\right) \xi-\left(\lambda_{2} d /\left(\lambda_{1}-\lambda_{2}\right)\right) \eta+\lambda_{2} z .
\end{aligned}
$$

Eliminating $t$,

$$
\begin{aligned}
& d \xi / d \eta=\left(\lambda_{1} / \lambda_{2}\right)(\xi / \eta), \\
& d z / d \eta=\left(\lambda_{1} d / \lambda_{2}\left(\lambda_{1}-\lambda_{2}\right)\right)(\xi / \eta)-d /\left(\lambda_{1}-\lambda_{2}\right)+z / \eta .
\end{aligned}
$$

Solving the first of these and making use of the result in the second we obtain as complete integral

$$
\begin{gathered}
\xi=C \eta^{1 / \kappa} \\
z=\left(C \lambda_{1} d /\left(\lambda_{1}-\lambda_{2}\right)^{2}\right) \eta^{1 / \kappa}-\left(d /\left(\lambda_{1}-\lambda_{2}\right)\right) \eta \log \eta+D \eta \\
(C, D \text { arbitrary }) .
\end{gathered}
$$

As before noted, every surface (12) passes through the origin in $\xi, \eta, z$ space, which is also the origin in $x, y, z$ space. Different possibilities can arise according as $\kappa$ is $>0$ or $<0$, and according as $d$ is zero or not.

$d \neq 0, \kappa>0 . \quad$ Each of the surfaces (24) contains the $\xi$-axis as a "stop line," since $\eta$ must be $\geqq 0$; and each of these

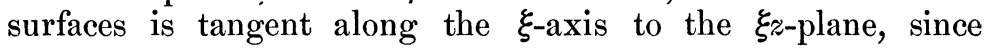


228 Singular points of a DifFerential EqUation. [Feb.,

$d z / d \eta=\infty$ for $\eta=0$. Hence all the $\infty^{2}$ integral curves enter $(0,0,0)$ in the half space $\eta \geqq 0$. This singular point is of the sort which Hilbert calls Endpunkt.

$d \neq 0, \kappa<0$. Write (12), (24) in the following form

$$
\xi \eta^{-1 / \kappa}=C
$$

$$
z \eta^{-1 / \kappa}=C \lambda_{1} d /\left(\lambda_{1}-\lambda_{2}\right)^{2}-\left(d /\left(\lambda_{1}-\lambda_{2}\right)\right) \eta^{1-1 / \kappa} \cdot \log \eta+D \eta^{1-1 / \kappa}
$$

For $C=0$ (12) gives the planes $\xi=0, \eta=0$, while (24) gives $\infty^{1}$ cylinders in space $\eta \geqq 0$ and having the $\xi$-axis as "stop line." The intersections of (12) and (24) therefore furnish, for $C=0, \infty^{1}$ curves containing $(0,0,0)$, all but one of them lying in the half-plane $\xi=0 \eta \geqq 0$ and having $(0,0$, $0)$ as "stop point," while that exceptional one is the $\xi$-axis itself.

For $C \neq 0,(12)$ gives a set of $\infty^{1}$ hyperbolic cylinders with generators parallel to the $z$-axis, while (24) gives (for $\eta$ small but $\nless 0$ ) a set of $\infty^{2}$ hyperbolic half-cylinders with generators parallel to the $\xi$-axis. The intersections of (12) and (24), therefore, furnish, for $C \neq 0, \infty^{2}$ curves all lying in space $\eta>0$; and none of these contains $(0,0,0)$. The singular point for $d \neq 0, \kappa<0$ is thus of a kind distinct from those previously considered. Only one integral curve passes through $(0,0,0)$; $\infty^{1}$ integral curves have $(0,0,0)$ as "stop point ;" and $\infty^{2}$ integral curves pass by $(0,0,0)$ in arbitrary proximity. This singular point might be call Sattel-Endpunkt mit isoliertem Strahl.

$d=0, \kappa \neq 0$. Our integrals now take the form

$$
\eta=C \xi^{\kappa}, \quad z=D \eta
$$

We have here no new kind of singular point. If $\kappa>0$, the origin is a Scheitelpunkt. If $\kappa<0$, the origin is a Sattelpunkt.

III II $_{\text {II }} \lambda_{1}=\lambda_{2}=\lambda_{3} \neq 0$. The three equations (10) now shrink to one, and we must seek still another means of integrating (4). Assume

$$
d x / d t=\alpha x+\beta \eta+\gamma z, \quad d \eta / d t=\lambda \eta, \quad d z / d t=\alpha^{\prime} x+\beta^{\prime} \eta+\gamma^{\prime} z
$$

where $\alpha, \beta, \gamma, \alpha^{\prime}, \beta^{\prime}, \gamma^{\prime}$ are to be determined so that the equations (26) shall be identities in $x, y, z$. The appropriate values of these constants are 


$$
\begin{array}{cc}
\alpha=\lambda^{2}(a-\lambda) /\left(\lambda^{2}+e\right), & \beta=b d /\left(\lambda^{2}+e\right), \\
& \gamma=\left(c \lambda^{2}-b \lambda+a b+c e\right) /\left(\lambda^{2}+e\right), \\
\alpha^{\prime}=\lambda^{2} d /\left(\lambda^{2}+e\right), & \beta^{\prime}=e d /\left(\lambda^{2}+e\right), \\
& \gamma^{\prime}=\lambda(f \lambda+2 e) /\left(\lambda^{2}+e\right) .
\end{array}
$$

We may now write (26) in the form

$$
\begin{aligned}
& d x / d \eta=\alpha x / \lambda \eta+\gamma z / \lambda \eta+\beta / \lambda, \\
& d z / d \eta=\alpha^{\prime} x / \lambda \eta+\gamma^{\prime} z / \lambda \eta+\beta^{\prime} / \lambda .
\end{aligned}
$$

From this we can pass, by the substitution $u=x+(\lambda-\alpha) z / \alpha^{\prime}$, to the equivalent pair

$$
\begin{aligned}
& d u / d \eta=u / \eta+\left(\lambda^{2}+e\right) / \lambda^{2}, \\
& d z / d \eta=\lambda d u /\left(\lambda^{2}+e\right) \eta+z / \eta+e d / \lambda\left(\lambda^{2}+e\right) .
\end{aligned}
$$

The integrals of (28) are

$$
u=\eta \log \left(C \eta^{1+e / \lambda^{2}}\right)
$$

(30) $z=(d / \lambda)\left[\left(e+\lambda^{2} \log C\right) \eta \log \eta /\left(e+\lambda^{2}\right)+\frac{1}{2} \eta(\log \eta)^{2}\right]+D \eta$.

By all the substitutions above used, the origin has remained unchanged, so that in $u, \eta, z$ space the critical point is $\operatorname{still}(0,0,0)$. In (29) $\eta$ may take negative as well as positive values, but not for the same value of $C$; so that the $z$ axis is a "stop line" for every surface represented by (29). In (30), however, $\eta$ must be $\geqq 0$ as long as $d \neq 0$. We must therefore distinguish two possibilities, according as $d$ is, or is not, zero.

$d \neq 0$. All the surfaces (29) will enter the $z$-axis tangent to the plane $\eta=0$ except when $1+e / \lambda^{2}=0$; but this exception may be excluded, since the above transformations would then be impossible. Every surface (30) contains the $u$-axis as a "stop line" and enters that line tangent to the plane $\eta=0$. The peculiarity of the singular point $(0,0,0)$ in the present case is that every curve of intersection of (29) and (30) not only enters it as a "stop point," but enters it from the same octant. The point might still be called an Endpunkt.

$d=0$. The surfaces (30) are now a one-parameter set of planes through the $u$-axis. All the integral curves have $(0,0,0)$ as a "stop point," which is therefore to be classed as Endpunkt.

ZüRICH, August 14, 1907. 